\title{
L'«Arqueta catalana» de la Biblioteca Reial
}

\author{
The «Catalan Ark» in the Royal Library
}

\author{
NARCÍs GAROLERA \\ Universitat Pompeu Fabra \\ narcis.garolera@upf.edu
}

Recibido: noviembre de 2020. Aceptado: diciembre de 2020

\begin{abstract}
Resum: L'article relaciona els títols dels llibres d'escriptors catalans conservats a l'Arqueta catalana de la Biblioteca Reial del Palacio de Oriente, de Madrid. S'hi transcriuen les dedicatòries dels autors - en català, la major part d'elles_-, entre els quals destaca, pel nombre de volums, Jacint Verdaguer. La destinatària habitual de les obres és la reina regent Maria Cristina, un retrat de la qual presideix l'esmentada arqueta.
\end{abstract}

Mots clau: escriptors, Arqueta, català, María Cristina, Renaixença

\begin{abstract}
The article lists the titles of books by Catalan writers preserved in the Catalan Ark of the Royal Library of the Palacio de Oriente in Madrid. The dedications of the authors are transcribed -in most of them in Catalan-, among which Jacint Verdaguer stands out for the number of volumes. The usual recipient of the works is Queen Regent Maria Cristina, a portrait of which presides over the aforementioned casket.
\end{abstract}

Keywords: writers, Arqueta, catalan, Maria Cristina, Renaixença.

A la Real Biblioteca del Palacio de Oriente, de Madrid, es conserva una col-lecció d'una trentena d'obres d'escriptors catalans i mallorquins del segle XIX, escrites en català, la majoria d'elles dedicades a la reina Maria Cristina d'Habsburg-Lorena, ${ }^{1}$ arxiduquessa d'Àustria, comtessa de Barcelona, i a les infantes Isabel de Borbón ${ }^{2}$ i María de la Paz de Borbón y Borbón. ${ }^{3}$

1 Maria Cristina d'Habsburg-Lorena (o d'Àustria) (Gross-Seelowitz, 1858 - Madrid, 1929), comtessa de Barcelona i segona esposa d'Alfons XII. A la mort d'aquest (1885) fou reina regent d'Espanya fins a la majoria d'edat del seu fill Alfons XIII (1902). Verdaguer hi tingué tracte personal.

2 Maria Isabel d'Espanya (Madrid, 1851 - París, 1931), primogènita d'Isabel II i Francesc d'Assís de Borbó, fou princesa d'Astúries en dues etapes (1851-57 i 1875-80).

3 Maria de la Pau d'Espanya, princesa de Baviera (Madrid, 1862 - Munic, 1946). El 1883 es casà amb el príncep Lluís Ferran de Baviera — cosí seu—, i visqué sempre més fora d'Espanya. Verdaguer li dedicà el poema «Enyorança» del recull Pàtria (1888). 
Segons el catàleg de la biblioteca, l'Arqueta catalana és una «Colección de obras de autores catalanes encuadernadas en tafilete rojo que se conservan en el interior de un mueble-librería en forma de arqueta de madera con herrajes metálicos; la llave y la cerradura reproducen los escudos del Condado de Barcelona y del Imperio austriaco; exterior forrado en terciopelo carmesí; interior de la tapa forrado en seda amarilla y terciopelo rojo que reproduce la señera».

Els llibres que guarda l'arqueta són dels anys 1860-1890. N'hi ha un d'anterior ${ }^{4} \mathrm{i}$ un de posterior, ${ }^{5}$ però tots els altres són obres impreses en aquest període. Hi destaquen, pel nombre — una dotzena—, les obres de Jacint Verdaguer, la majoria dedicades en català. N'hi ha d'altres autors vigatans, com Jaume Collell, Martí Genís i Joaquim Salarich.

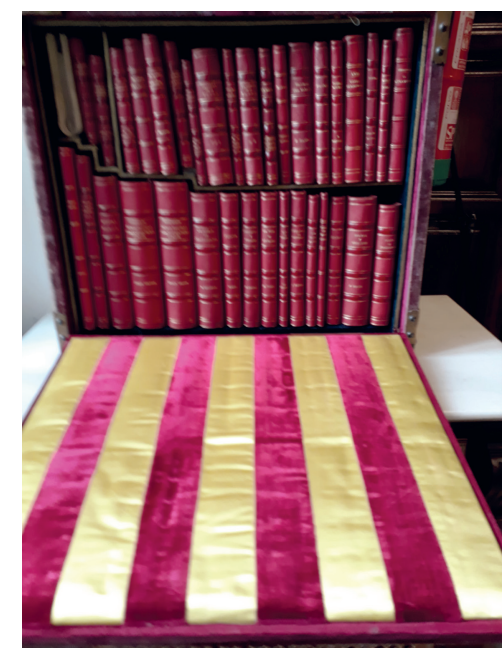

INTERIOR DE L'ARQUETA CATALANA

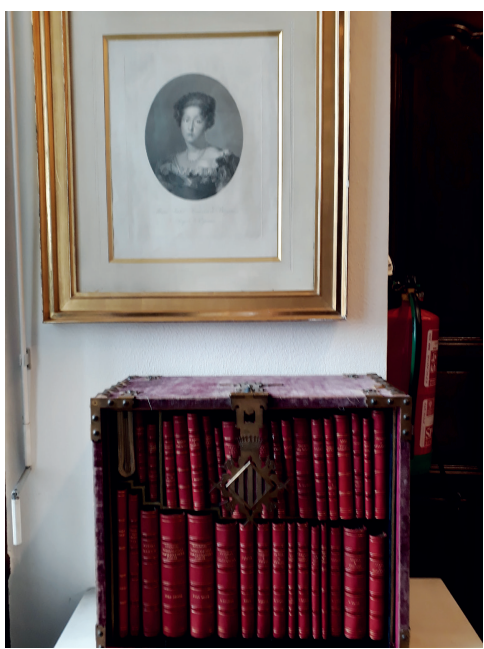

ARQUETA I RETRAT DE MARIA CRISTINA

Hi ha llibres de noms destacats de la Renaixença literària, com Manuel Milà i Fontanals, Joaquim Rubió i Ors, Víctor Balaguer, Frederic Soler i Francesc Pelagi Briz. La representació mallorquina la formen Bartomeu Ferrà i Jeroni Rosselló.

És destacable que pràcticament tots els volums dedicats ho són a la reina Maria Cristina, anomenada per molts escriptors «Arxiduquesa d'Austria» i «Comptesa de Barcelona». ${ }^{6}$ I on podem detectar un "austriacisme" explícit és en una dedicatòria de Jaume Collell a la reina:

\footnotetext{
${ }^{4}$ La segona edició de les poesies del Gayter del Llobregat —Joaquim Rubió i Ors- és del 1858 , però la tramesa i la dedicatòria són força posteriors.

5 Flors de Maria (1902), de Jacint Verdaguer, fou enviat a la reina regent poc abans de la mort del poeta.

${ }^{6}$ Maria Cristina presidí, com a reina regent d'Espanya, els Jocs Florals de Barcelona del 1888.
} 
«Al Real brot de la gran Casa d'Austria / los nets dels Vigatans».7

La major part de dedicatòries són escrites en català, i una, adreçada a la reina, ho és en alemany, ${ }^{8}$ en lletra gòtica, molt difícil de llegir.

La disposició dels títols, alfabètica, és per autors. En el cas d'obres anònimes, l'entrada és pel títol. La descripció bibliogràfica és sumària: autor, títol, lloc d'edició, editorial i data de publicació. Si el llibre du una dedicatòria la transcric literalment, mantenint la disposició gràfica de l'original autògraf.

Vull expressar el meu agraïment al personal de la Real Biblioteca de Palacio per l'interès amb què van atendre les meves peticions $\mathrm{i}$ per les facilitats concedides per a la reproducció de les portades i les dedicatòries dels llibres de l'Arqueta catalana.

$$
* * *
$$

Ramon Arabía y Solanas: ${ }^{9}$ "Los Clubs Alpins y las Associacions d' Excursions». Conferencia donada [...] en la Associació d' Excursions Catalana per D. -----. Barcelona: Imprenta de la Renaixensa, 1879.

L'exemplar és dedicat a la reina Maria Cristina:

[...] Majestät der Königin von Spanien, Erzherzogin von Osterreich [...]

Präsident der Catalanischen Excursions Verein, Mitglied von Französischen Allg[emein]en Clubs und von [...] Clubs.

Barcelona den 29 Marz. 1879.

Agnés Armengol de BadíA: ${ }^{10}$ Lays de ------. Barcelona: Tipografía Espanyola, 1879. [Retrat de l'autora, sota del qual hi ha la seguent dedicatòria:]

A sa A[ltesa] R[eal] é I[lustríssima] la Comptesa de Barcelona, Na Maria Cristina de Habsburg.

Agnés Armengol

7 Durant la Guerra de Successió Hispànica (1702-1715) els partidaris de l'arxiduc Carles d'Àustria eren coneguts, genèricament, com a "vigatans". d'Àustria.

8 L'alemany era la llengua materna de Maria Cristina d'Habsburg-Lorena, arxiduquessa

9 Mataró, 1850 - Barcelona, 1902. Promotor de l'excursionisme català, i partidari de les relacions amb clubs alpins estrangers, presidí (1879-84) l'Associació Catalana d'Excursions, escissió de l'Associació Catalanista d'Excursions Científiques.

10 Sabadell, 1852-1934. Escriví poesia de caire molt tradicional. 
Joseph María Arnau: ${ }^{11}$ ¡Donas! Comedia en tres actes y en vers, original de D. ---. Barcelona: Llibrería de Eudalt Puig, 1875.
A S. A. la Reyna de España
Comptesa de Barcelona
$\mathrm{B}$ [esa] S[os] R[eials] P[eus]
L' Autor.

Víctor Balaguer: ${ }^{12}$ Trajedias. Su autor en verso catalán ----- [...] Segunda edición. Madrid: Imprenta de Fortanet, 1878.

MARÍA DE BELL-LLOCH: ${ }^{13}$

Poesías catalanas ab un prólech de Joan Sardá. Barcelona: Estampa de la Renaxensa, 1874.
A S. M. Na Maria Cristina Reyna de Espanya
Maria de Bell-lloch
Barcelona 7 Febrer de 1880

Vigatans y botiflers. Novela histórica per D. ${ }^{\mathrm{a}}$-----. Barcelona: Joan Roca y Bros, 1878.

Quan de temps vos so esperada,

Oh Regina y gran Senyora!

Mes al fi ja ha arrivat l'hora

Pera mi tan desitjada.

Deu vos donga bona estada

En ma terra que us adora.

$\mathrm{M}^{\mathrm{a}}$ de Bell-lloch

Barcelona 7 Febrer de $1880^{14}$

\section{Francesch Pelay Briz: ${ }^{15}$}

Cansons de la terra. Cants populars catalans col-leccionats per ----- y Candi Candi. Barcelona: Llibreteria de E. Ferrando Roca, 1866.

11 Josep Maria Arnau i Pascual (Arenys, 1831-1913). Comediògraf costumista.

12 Víctor Balaguer i Cirera (Barcelona, 1824 - Madrid, 1901). Polític, historiador, poeta i dramaturg.

13 Pseudònim de Maria del Pilar Maspons i Labrós (Barcelona, 1841-1907), germana del folklorista Francesc Maspons i cunyada de Francesc Pelagi Briz.

${ }_{14}$ Primer viatge de la reina a Barcelona, poc després de casar-se amb Alfons XII (1879).

15 Francesc Pelagi Briz i Fernández (Barcelona, 1839-1889). Escriptor romàntic, editor i folklorista. 
Ofereix aquest llibre
A S. A. R. é I. Na Maria Cristina
Arxiduquesa d' Austria
$\mathrm{y}$
Reyna d' Espanya
en penyora de respetuosa adhesiò
Francesch Pelay Briz

Cansons de la terra. Cants populars catalans. Col-lecció premiada en la Exposició de Viena de 1873 y publicada per -----. Volum quart. Barcelona: Alvar Verdaguer I Paris: Maisonneuve \& C. ${ }^{\mathrm{e}}, 1874$.

\section{A S. M. Na Maria Cristina \\ Arxiduquesa d' Austria.}

Senyora, d'est volum á dintre hi ha tancadas del poble catalá las joyas mes preuhadas, sas cansons, sas costums, de son parlar la flor: Si mai sentiu del dol las greus agullonadas, obríulo é hi trobaréu melódicas tonadas que tornaran la pau á vostre noble cor.

Francesch Pelay Briz

Antoni Careta y Vidal: ${ }^{16}$ Brosta. Qüentos, escenas de costums, tradicions, novelas, fantasias, per -----. Barcelona: Joan Roca y Bros, 1878.

Á S[a] A[ltesa] R[eial] é I[lustríssima]

Na Maria Cristina, Arxiduquesa d' Austria,

Princesa de Catalunya, Comptesa de Barcelona.

\section{Endreça}

Ves, llibre; y si, algun dia, eixint de l' artisada llibreria, desclou tos fulls sa delicada má, digas: «Só lo recort d' un' anyoransa, só penyora d' amor y d' esperansa, m’ envia un catalá!»

Antoni Careta y Vidal

16 Barcelona, 1843-1924. Narrador costumista i comediògraf. 
Jaume Collell: $:^{17}$ Poesias de D. ----- premiadas en los Jochs Florals de Barcelona del any 1869. [...] Barcelona: Estampa de Celestí Verdaguer, 1869.

Compendi historial de la Biblia que ab lo títol de Genesi de Scriptura trelladá del provençal a la llengua catalana Mossen Guillem Serra ${ }^{18}$ en l' any M.CCCCLI, y ara ha fet estampar per primera vegada En Miquel Victoriá Amer. Barcelona: Any MDCCCLXXIII.

Lluís Cutchet: ${ }^{19}$ Historia del siti de Girona en 1809. Barcelona: Aleix Sierra, 1868.

\author{
A S. M. la Reyna \\ María Cristina \\ L'Autor [rubricat]
}

Discursos leídos ante la Real Academia de la Historia en la recepción pública del Excmo. Señor Don Víctor Balaguer [...]. Barcelona: Imprenta de Narciso Ramírez y C. ${ }^{a}, 1876$.

Ofert á S. M. la Regina Na Maria Cristina, Comptesa de Barcelona, per

Domingo Mas.

Diccionari Catalá-Castellá-Llatí-Frances-Italiá. Per una societat de catalans. Tomo Primer. Barcelona: Imprempta de Joseph Torner, 1839.

Diccionari Catalá-Castellá-Llatí-Frances-Italiá. Per una Societat de Catalans. Tomo Segòn. Barcelona: Imprempta de Joseph Torner, 1839.

Bartomeu Ferrá: ${ }^{20}$ Comèdies y poesies de En -----. Palma: Estampa d'en Felip Guasch, 1872.

La garba montanyesa. Recull de poesies del Esbart de Vich. Vich: Estampa y llibreria de Ramon Anglada, 1879.

Al Real brot de la gran Casa d'Austria los nets dels Vigatans. ${ }^{21}$

17 Jaume Collell i Bancells (Vic, 1846-1932). Eclesiàstic i escriptor. Periodista polèmic, fundà i dirigí el setmanari La Veu del Montserrat.

18 Guillem Serra (s. XV), cavaller.

19 Lluís Cutchet i Font (Llívia, 1815 - Barcelona, 1892). Periodista, historiador i polític liberal.

${ }^{20}$ Bartomeu Ferrà i Perelló (Palma, Mallorca, 1843-1924). Mestre d'obres, escriptor i arqueòleg.

${ }^{21}$ La dedicatòria no va signada, però la lletra és de Collell, compilador del recull. 
Martí Genís y Aguilar:22 Julita, per -----. Barcelona: Imprenta de la Renaxensa, 1874.

\author{
A S. M. \\ $D^{a}$ Maria Cristina d'Austria \\ Reina d' Espanya
}

L'autor

Homenage al beato Raimundo Lull en el sexto centenario de la fundación del Colegio de Miramar. Palma: Establecimiento Tipográfico de Pedro José Gelabert, 1877.

\author{
Ofereix aquest llibre \\ A S. M. la Reyna Na María Cristina, \\ Arxiduquesa d' Austria, \\ en penyora de leal y respectuosa adhessiò \\ Francesch Pelay Briz.
}

Libre del romiatge d[e]l Venturos Pelegri, ab les Cobles de la Mort [...]. S. 1., s. d. $[1879]{ }^{23}$

Ausias March,${ }^{24}$ Obras de aquest poéta publicadas [...] per Francesch Pelayo Briz [...]. Barcelona: Llibreteria de E. Ferrando Roca, 1864.
A S. M. Na Maria Cristina
Reyna d' Espanya
$\mathrm{y}$
Comptesa de Barcelona
Laura inspirá á Petrarca ab sa bellesa cants d' hont ne vessan armonia y mel.
A nostre Ausías inspirá, Teresa, cants que cautivan com baixats del cel.
Pro son uns de gaya fantasía,
los altres clams d' agegantat dolor;

22 Vic, 1847-1932. Poeta i narrador. Julita és la seva obra més reeixida.

23 A la Biblioteca consta com a part del Cançoner de les obretes en nostra lengua materna mes divulgades durant los segles XIV, XV e XVI, compilada per Marian Aguiló «entre els anys 1873-1900, en diversos fascicles tirats en lletra gòtica amb profusió de gravats i ornaments» (Joan Mahiques Climent, «Sobre la difusió de les Cobles de la Mort al segle XVI», Catalan Review, XXXI (2017), p. 52.

24 Ausias (o Ausiàs) March (València, 1400-1459). Poeta i cortesà. La seva obra es compon de 128 poemes, conservats en tretze manuscrits de cap a la fi del segle XV i de la primera meitat del XVI. 
ab l' art, Petrarca, los seus lays vestía,

March en los seus hi va posá 'l seu cor.

Si Vos, Senyora, los llegiu un día,

ben cert ne so, direu ab melangía:

"Petrarca es plata; mes, Ausías, or".

\section{Francesch Pelay Briz}

Francisco Maspons y Labrós ${ }^{25}$ [...]: Tradicions del Vallés per -----. Barcelona: Estampa de «La Renaxensa», 1876.

A sa A. R. é I.

$\mathrm{D}^{\mathrm{a}}$ Maria Cristina, Arxiduquesa

d'Austria, Reyna d'Espanya

lo colector de la tradició catalana

F. Maspons y Labrós

Artur MasRiera y Colomer: ${ }^{26}$ Poesías líricas (históricas, bíblicas y populars) per -----. Barcelona: Llibrería d' Alvar Verdaguer, 1879.

A la Comtesa de Barcelona:

A. Masriera [rubricat]

1880 .

Manel Milá y Fontanals: ${ }^{27}$ La cansó del pros Bernart fill de Ramon feta per en ----- lo mes de juny de l'any de la Nativitat del Senyor MDCCCLXVII. [Barcelona: Estampa d'en Celestí Verdaguer, 1867]

A S. M. Na Maria Cristina

ofereix respectuosament sa cansó

Lo vell juglar [rubricat]

${ }^{25}$ Francesc de Sales Maspons i Labrós (Granollers, 1840 - Bigues, 1901). Folklorista de ressò europeu.

26 Barcelona, 1860-1929. Poeta, narrador i publicista.

27 Manuel Milà i Fontanals (Vilafranca del Penedès, 1818-1884). Filòleg i escriptor. Catedràtic de literatura de la Universitat de Barcelona, exercí un magisteri fecund. Presidí els primers Jocs Florals de Barcelona (1859). 


\section{Dolors Monserdá de Maciá:28}

Discurs de D. ${ }^{a}$----- llegit en lo Saló de Cent de la casa Consistorial [...] en las festas y firas de la verge de las Mercés de 1879. Barcelona: Imprenta de la Renaxensa, 1879.

Poesies catalanes de ------. Barcelona: Imprenta de la Renaxensa, 1888.

Victoria Penya D’Amer: ${ }^{29}$ Una visita á ma Patria, poesia de $\mathrm{Na}$-----, qui guanyá primer accéssit á la Flor natural y premi extraordinari d' una Rosa d' argent en los Jochs Florals de MDCCCLXXIII. [Barcelona: Estampa de C. Verdaguer y $\mathrm{C}^{\mathrm{a}}, 1873$ ]

SERAfí PitARRA: ${ }^{30}$ Lo Rector de Vallfogona. Drama en tres actes y en vers, original de D. ----- (Frederich Soler) [...]. Segona edició. Barcelona: Llibrería d' Eudalt Puig, 1874.

Geróni Rosselló: ${ }^{31}$ Lo joglar de Maylorcha per ----- Mestre en Gay Saber. Palma: Estampa de Pere Joseph Gelabert, 1862.

Johquim Rubió y Ors: ${ }^{32}$ Lo Gayter del Llobregat. Poesias de D. -----. Segona edició [...]. Barcelona: Libreria de Joseph Rubió, 1858.

Puesto con el mayor respeto á sus reales pies, ofrece a S. M. la Reyna Doña Maria Cristina, rogándole humildemente que se sirva aceptarlas, estas modetísimas producciones poéticas, frutos primerizos en su mayor parte de su escaso ingenio, y tributo por demás pobre para quien, de tantas y tan relevantes prendas está adornada,

Joaquin Rubió y Ors.

28 Dolors Monserdà i Vidal (1845-1919). Poeta i novel-lista, autora d'obres de caràcter social i feminista.

29 Victòria Peña i Nicolau (Palma, Mallorca, 1827 - Barcelona, 1898). Poeta influïda per la lírica popular. Germana de l'escriptor Pere d'Alcàntara Peña i muller del poeta Miquel Victorià Amer.

30 Pseudònim de Frederic Soler i Hubert (Barcelona, 1839-1895). Comediògraf, dramaturg i poeta.

31 Jeroni Rosselló i Ribera (Palma, Mallorca, 1827-1902). Poeta i lul-lista. Usà un llenguatge arcaic, sobretot en composicions sobre la història de Mallorca.

${ }^{32}$ Barcelona, 1818-1899. Escriptor i erudit. Fou catedràtic de literatura de la Universitat de Barcelona, i impulsor de la restauració dels Jocs Florals. 
JoAquim Salarich: ${ }^{33}$ Lo castell de Sabassona. Narració de costums de la Edat Mitjana, per D. ----- [...]. Vich: Estampa y llibreria de R[amon] Anglada, 1879.

\author{
A S. M. D ${ }^{a}$ Maria Cristina d' Austria \\ Reyna d' Espanya
}

Li suplica se digne acceptar aquesta petita ofrena

\title{
L' Autor.
}

Eduart Toda y GüEll: $:^{34}$ Poblet. Recorts de la Conca de Barberá. Barcelona: [Estampa de la Renaxensa], 1883. ${ }^{35}$

JACINTO VERDAGUER: ${ }^{36}$

La Atlántida. Poema de ----- ab la traducció castellana per Melcior de Palau. Barcelona: Estampa de Jaume Jepús, 1878.

A S. M. el Rey D. Alfonso XII. ${ }^{37}$

De mar en mar, de terra en terra, un dia

aquest ramell de tradicions cullia

d'una nació gegant.

Vos que 'n sou Rey, rebeu l'oferta mia;

lo ram es xich, peró l'afecte es gran.

Jacinto Verdaguer Pbre. ${ }^{38}$

La Atlántida. Poema de ----- ab la traducció castellana per Melcior de Palau. Barcelona: Estampa de Jaume Jepús, 1878.

A S. M.

$\mathrm{D}^{\mathrm{a}}$ María Cristina d'Austria,

Reyna d'Espanya,

Comptesa de Barcelona,

L Autor.

33 Joaquim Salarich i Verdaguer (Vic, 1816-1884). Metge, historiador i escriptor. Cronista de la ciutat de Vic, publicà diverses obres sobre la ciutat. Autor de novel-les i narracions de caràcter romàntic.

${ }^{34}$ Eduard Toda i Güell (1855 - Poblet, 1941). Diplomàtic i escriptor.

35 A la portadella, segell de l'Associació Catalanista d'Excursions Científiques.

36 Jacint Verdaguer i Santaló (Folgueroles, 1845 - Vallvidrera, 1902). Poeta. Representant màxim de la Renaixença, consolidà la literatura en català. L'Atlàntida (1877) i Canigó (1886), traduïdes a diverses llengüies, li donaren fama internacional. Autor també, en prosa modèlica, de llibres de viatges.

37 Alfons XII d'Espanya (Madrid, 1857 - El Pardo, 1885). Fill d'Isabel II i de Francesc d'Assís de Borbó. El març del 1885 rebé amb simpatia el «Memorial de Greuges», però no rectificà la política centralista de Cánovas del Castillo. Verdaguer hi tingué tracte personal.

38 Aquesta i les altres dedicatòries foren recollides a Marta Pessarrodona - Narcís Garolera, Jacint Verdaguer. Una biografia (Barcelona: Quaderns Crema, 2016), p. 286-289. 
Idilis y cants mistichs, per -----, ab un prolech de D. M. Milá y Fontanals. Barcelona: Llibreria d'Eusebi Riera, 1879.

\author{
A S. M. \\ $\mathrm{D}^{\mathrm{a}}$ María Cristina d'Austria, \\ Reyna d'Espanya
}

1 Autor.

Canigó. Llegenda pirenayca del temps de la Reconquista, per -----. Barcelona: Llibreria Católica, 1886.

\author{
A sa Magestat \\ $\mathrm{D}^{\mathrm{a}}$ Maria Cristina, Reyna \\ Regent d'Espanya,
}

Jacinto Verdaguer Pbre.

Lo somni de Sant Joan. Llegenda del Sagrat Cor de Jesús per -----, ab la traducció castellana. Barcelona: Llibrería y Tip[ografia] Católica, 1887.

\author{
A S. M. \\ $\mathrm{D}^{\mathrm{a}}$ Maria Cristina \\ Reyna Regent d'Espanya. \\ Homenatge de respecte y admiració, \\ Jacinto Verdaguer Pbre. [rubricat]
}

Flors de Maria, per -----. Barcelona: Estampa de la Casa P. de Caritat, 1902.

Al depositar á los R[eales] P[ies] de S. M., que se acordó piadosamente del enfermo desvalido, esta humildisima ofrenda, fervorosamente ruego á Dios supla su ningun valor derramando sobre $S$. $M$. y la Real Familia toda suerte de prosperidades.

[Escrit d'una altra mà]

J. Verdaguer Pro.

[Signatura autògrafa $]^{39}$

${ }^{39}$ El 7 d'abril un agutzil s'havia personat al pis del carrer d'Aragó on vivia Verdaguer, ja malalt, per cobrar les costes del judici — promogut i perdut - contra el Dr. Pere Manaut, que l'havia declarat boig. Les costes pujaven a 1001 pessetes (3503,50 euros). El 23 d'abril la reina regent féu arribar a Verdaguer 1000 pessetes (3500 euros), que li serviren per pagar —o compensar- aquelles costes. D'aquí la tramesa del llibre, aparegut feia poc, i la dedicatòria —d'una altra mà- a la reina per agrair-li l'ajut econòmic. 
La Atlántida. Poema de ----- ab la traducció castellana per Melcior de Palau. Barcelona: Estampa de Jaume Jepús, 1878.

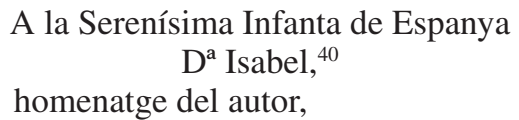

Jacinto Verdaguer Pbre.

Idilis y cants mistichs per -----, ab un prolech de D. M. Milá y Fontanals. Barcelona: Llibreria d'Eusebi Riera, 1879.
A la Serenísima Infanta de España,
$\mathrm{D}^{\mathrm{a}}$ Isabel, en testimonio de adhesion y reconocimiento, Jacinto Verdaguer Pbro.

Cansons de Montserrat i Llegenda de Montserrat per ----- [en un volum]. Vich: Estampa de Ramon Anglada, 1880.

A la Serenísima Infanta de España,

$\mathrm{D}^{\mathrm{a}}$ Isabel, en testimonio de adhesion y reconocimiento, Jacinto Verdaguer Pbro.

Comillas, dia de la Coronacion de la Virgen de Montserrat.

Canigó. Llegenda pirenayca del temps de la Reconquista, per -----. Barcelona: Llibreria Católica, 1886.

$$
\text { A S. R. A. }
$$

$\mathrm{D}^{\mathrm{a}}$ Isabel de Borbon,

Infanta de España,

$$
\text { Jacinto Verdaguer Pbro. }
$$

Idilis y cants mistichs per -----, ab un prolech de D. M. Milá y Fontanals. Barcelona: Llibreria d'Eusebi Riera, 1879.

A la Serenísima Infanta de España,

$\mathrm{D}^{\mathrm{a}}$ María de la Paz, ${ }^{41}$

humilde homenaje del

Autor.

40 Maria Isabel d'Espanya. Vegeu la nota 2.

41 Vegeu la nota 3. 
Nazareth per Mossen -----. Barcelona: [Librería de Bastinos], 1890.
A Sa Altesa
la $S^{a} D^{a}$ Pau de Borbon
Princesa de Baviera,
ab qui visca eternament
lo bon Jesus.
Jacinto Verdaguer Pbre.
Barcelona, Pasqua de Resurrecció de 1890.

\section{CONCLUSIONS}

La present relació bibliogràfica il-lustra l'interès dels escriptors catalans del segle XIX per la monarquia espanyola de la Restauració, sobretot envers la persona de la reina Maria Cristina d'Habsburg-Lorena, arxiduquessa d'Àustria i comtessa de Barcelona, motius pels quals la major part de les dedicatòries li són adreçades. En la de Jaume Collell a un volum de poesies és evident l'austriacisme dels descendents dels "vigatans", partidaris de la casa d'Àustria durant la Guerra de Successió a la corona hispànica. I recordem que, el maig del 1888, la reina presidí els Jocs Florals de Barcelona, on el polígraf Marcelino Menéndez y Pelayo - format a la capital de Catalunya — pronuncià un discurs en llengua catalana.

És destacable l'ús del català en les dedicatòries —algunes de les quals són en vers-, i també que la majoria d'obres trameses són escrites en aquesta llengua.

Jacint Verdaguer és l'autor que més obres seves adreça a membres de Palau: el rei Alfons XII, la reina Maria Cristina, les infantes Isabel i Maria de la Pau, a qui Verdaguer dedicà també una poesia - "Enyorança"— amb motiu del seu casament amb el príncep Ferran de Baviera, que li suposà viure sempre més allunyada de la Cort espanyola.

En resum, la relació bibliogràfica que presento permet comprovar l'interès d'un nombre representatiu d'escriptors catalans de la Renaixença per alguns dels membres més destacats de la reialesa espanyola de la segona meitat del segle XIX. 